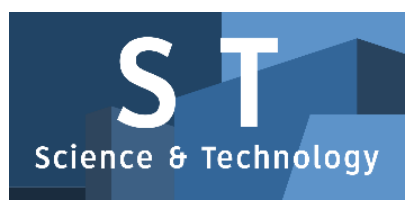

PAPER - OPEN ACCESS

\title{
The Other Assignment For Problem Instances Esc 16b, Esc 16c
} And Esc 16h

\author{
Author \\ : Faiz Ahyaningsih \\ DOI \\ : 10.32734/st.v1i1.188 \\ Electronic ISSN \\ : 2654-7088 \\ Print ISSN \\ : 2654-7080
}

Volume 1 Issue 1 - 2018 TALENTA Conference Series: Science \& Technology (ST)

\section{(ㄷ) $(1) \Theta$}

This work is licensed under a Creative Commons Attribution-NoDerivatives 4.0 International License.

Published under licence by TALENTA Publisher, Universitas Sumatera Utara
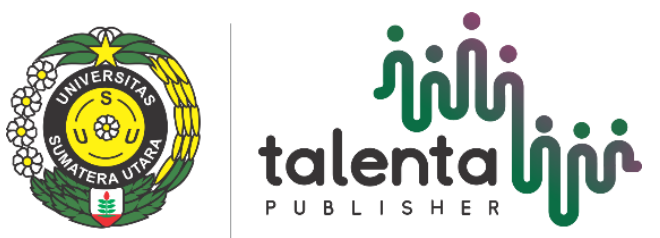


\title{
ijoli talentalioำ TALENTA Conference Series \\ P U B L I S H E R Available online at https://talentaconfseries.usu.ac.id \\ The Other Assignment For Problem Instances Esc 16b, Esc 16c And Esc $16 \mathrm{~h}$
}

\author{
Faiz Ahyaningsih \\ Mathematic Department, Universitas Negeri Medan, Medan-20220, Indonesia \\ faizahyaningsih@yahoo.com
}

\begin{abstract}
The quadratic assigment problem (QAP) has remainedone of the great challenges in combinatorial optimization. In this paper I propose two programs, the MATLAB program for solving QAP, and the MATLAB program for checking objective value, if we input an arbitrary permutation, matrix flow and matrix distance. The first program using combination methods that combines random point strategy, forward exchange strategy, and backward exchange strategy. I've tried my program to solve Esc 16b, Esc $16 \mathrm{c}$ and Esc 16h from QAPLIB (A Quadratic Assignment Problem Library). In the 500th iteration optimal value reached and I've found the other assignment for problem instances Esc 16b, Esc 16c, and Esc 16h.
\end{abstract}

Keywords :Backward Exchange Strategy; Combination Methods; Forward Exchange Strategy; Quadratic Assigment Problem; Random Point Strategy

\section{Introduction}

The Quadratic assignment problem (QAP) has remained one of the great challenges in combinatorial optimization. It is still considered a computationally nontrivial task to solve modest size problems, say of size $n=20$. The QAPLIB provide a unified testbed for QAP, accessible to the scientific community, and consisted of virtually all QAP instances. In 1957 QAP was introduced by Koopmann and Beckmann as a mathematical model for the location of a set of indivisible economic activities. The QAP however is not only nonlinear but it is not unimodal. As a consequence this problem class has attracted active investigation by numerous researches. An heuristic yielding a good solution seems a more appropriate course of action than attempting an optimal solution, and many of the papers aim at achieving improvements to an initial assignment.

One of the oldest applications of the QAP is the assignment of specialized rooms in a building (Elshafei, 1977). In this case, aijis the flow of people that must go from service $\mathrm{i}$ to service $\mathrm{j}$ and bklis the time for going from room $\mathrm{k}$ to rooml. A more recent application is the assignment of gates to airplanes in an airport; in this case, aijis the number of passengers going from airplane $\mathrm{i}$ to airplane $\mathrm{j}$ (a special - airplane $\|$ is the main entrance of the airport) and bkl is the walking distance between gates $\mathrm{k}$ and 1 .

Even though QAP have investigated over 58 years ago, it remains one of the most inefficient for some problem instances, as we know from QAPLIB report.

Finally, let us mention other applications in imagery (Taillard, 1995), turbine runner balancing (Laporte and Mercure, 1998) and the fact thatproblems such as the travelling salesman or the linear ordering can be formulated as special QAPs. 


\section{Literature Review}

The quadratic assignment problem was one of the first problems solved by metaheuristics methods first conceived in the 1980's. Burkard and Rendl [6] proposed a simulated annealing procedure that was able to find much better solutions than all the previously designed heuristic methods. Six years later, Connolly (1990) proposed an improved annealing scheme. His method is easy to set up, since the user

has to select the number of iterations; and all other parameters are automatically computed. At around the same time, Skorin-Kapov [17] proposed a tabu search. Then, Taillard (1991) proposed a more robust tabu search, with fewer parameters and running $\mathrm{n}$ times faster than the previous implementation. Recently Ahyaningsih and Sitompul (2015) proposed a combined strategy for solving QAP.

Other tabu searches have been proposed, such as the reactive tabu search of Battiti and Tecchiolli (1994a). In the same year Battiti and Tecchioli (1994b) compared tabu search techniques with simulated annealing. and the star-shape diversification approach of Sondergeld and Voss (1996).

Genetic algorithms have also been proposed, for instance by Tate and Smith (1995),Misevicius and Rubliauskas (2009), but hybrid approaches, such as those of Fleurent and Ferland (1994), Ahuja et al. (2000), Drezner (2002a, 2002b), provides a good review for QAP.

Another heuristic, GRASP (greedy randomized adaptive search procedure) was proposed by Liet. al. (1994). An ant colony system approaches by Taillard, (1998), Gambardella et al., (1999), Stutzle and Hoos,(1999) have beenproposed, as well as a scatter search (Cung et al., 1997). Some of these methods have been compared in Taillard (1995) who showed that the efficiency of these methods strongly depends on the problem instance type to which they are applied. _Heuristic Kelayakan Methods' was proposed byAhyaningsih (2006).

\section{The Quadratic Assignment Problem}

This is combinatorial problem of deciding the placement of facilities in specified locations in such a way as to minimize a quadratic objective function. Consider the problem of locating $\mathrm{n}$ facilities in $\mathrm{n}$ given locations. If the flow $f_{i k}$ between each pair of facility $i$ and facility $k$ and the unit transportation cost (or distance) $d_{j l}$ between locations $j$ and 1 are known, then the problem is defined to be

$$
\begin{aligned}
& \operatorname{Min} \emptyset=1 / 2 \sum_{i=1}^{n} \sum_{k=1}^{n} \sum_{j=1}^{n} \sum_{l=1}^{n} f_{i k} d_{j l} x_{i j} x_{k l} \\
& \text { subject to } \sum_{j=1}^{n} x_{i j}=1, \mathrm{i}=1,2, \ldots, \mathrm{n} \\
& \sum_{i=1}^{n} x_{i j}=1, \mathrm{j}=1,2, \ldots, \mathrm{n} \\
& \quad 0 \leq x_{i j} \leq 1 \\
& x_{i j} \text { integer }
\end{aligned}
$$

Matrices $\left[f_{i k}\right]$ and $\left[d_{j l}\right]$ are assumed to be symmetric. The assignment variable $x_{i j}$ has a value 1 if facility $i$ at location $j$, and has a value 0 otherwise. The constraints reflect the fact that each location can be assigned to only one facility, and each facility can be assigned to only one location. 


\section{Strategy For Solving Qap}

\subsection{Random Point Strategy}

- Read $\theta=10000000$, matrix $\left[f_{i k}\right]$ dan $\left[d_{j l}\right], n=$ problem dimension and ITEMas the number of iteration, $a=1$ (initial iteration)

- Generate random permutation, $x_{\mathrm{i}}=\operatorname{randperm}(n)$; for every facility $i, i=1,2, \ldots, n$.

- $x_{i j}=1$ for gen $x_{i}=j ; x_{i j}=0$ for generate $x_{i} \neq j ; \mathrm{i}, \mathrm{j}=1,2, \ldots, \mathrm{n}$

- Calculate the objective function value

$$
\bar{\theta}=\sum_{i=1}^{n} \sum_{k=1}^{n} \sum_{j=1}^{n} \sum_{l=1}^{n} f_{i k} d_{j l} x_{i j} x_{k l}
$$

- if $\theta<\theta$, then $\theta=\theta ; \mathrm{a}=\mathrm{a}+1$

- if a $<$ ITEM, back to 2)

- STOP

\subsection{Forward Exchange Strategy}

1) Input $n$ as problem dimension.

2) Read flow matrix $f_{i k}$, distance matrix $d_{j e}$, initial asssignment $x_{i j}$.

3) Calculate

$$
\theta-\sum_{i=1}^{n} \sum_{k=1}^{n} \sum_{j=1}^{n} \sum_{i=1}^{n} f_{i k} d_{j k} x_{i j} x_{k t}
$$

4) Generate $x_{i}=j$ for $x_{i j}=1 ; i, j=1, \ldots, n$

5) $a=1$

6) $b=1$

7) $c=\operatorname{Gen} x_{i=a} ;$ Gen $x_{t=a}=\operatorname{Gen} x_{i=a+b} ; \operatorname{Gen} x_{\mathrm{i}=a+b}=c$

8) $x_{i j}=1$ for $\operatorname{Gen} x_{i}=j, x_{i j}=0$ for $\operatorname{Gen} x_{i} \neq j, i, j=1, \ldots, n$

9) Calculate objective function

$$
\bar{\theta}-\sum_{i=1}^{n} \sum_{k=1}^{n} \sum_{j=1}^{n} \sum_{j=1}^{n} f_{k k} d_{j k} x_{b j} x_{k}
$$

10) if $\bar{\theta}<\theta$ then $\theta=\bar{\theta}$ go to (14)

11) $\mathrm{c}=\operatorname{Gen} x_{i=a+b} ; \operatorname{Gen} x_{i=a+b}=\operatorname{Gen} x_{i=a} ; \operatorname{Gen} x_{i=a}=c$

12) $b=b+1$

13) if $b \leq n-a$ go to 7 )

14) $a=a+1$

15) if $a \leq n$ go to 6 )

16) STOP 


\subsection{3. Backward Exchange Strategy}

1) Input $n$ as problem dimension

2) Read flow matrix $f_{i k}$, distance matrix $d_{j e}$ initial assignment $x_{i j}$.

3) Calculate $\theta-\sum_{i=1}^{n} \sum_{k=1}^{n} \sum_{j=1}^{n} \sum_{k=1}^{n} f_{k} d_{j t} x_{y} x_{k}$

4) Gen $x_{1}=j$, for $x_{i j}=1 ; \mathrm{i}, j=1, \ldots, n$

5) $a=n$

6) $b=n$

7) $c=\mathrm{Gen} x_{i-a ;}$ Genx $x_{i-a}=\mathrm{Gen} x_{i=b} ; \operatorname{Gen} x_{i-b}=c$

8) $x_{i j}=1$ for $\operatorname{Gen} x_{i}=j, x_{i j}=0$ for $\operatorname{Gen} x_{i j} j, i, j=1, \ldots, n$

9) Calculate objective function

$$
\bar{\theta}-\sum_{i=1}^{n} \sum_{k=1}^{n} \sum_{j=1}^{n} \sum_{k=1}^{n} f_{k} d_{p} x_{\xi} x_{k}
$$

10) if $\bar{\theta}<\theta$ then $\theta=\bar{\theta}$ go to 14)

11) $\mathrm{c}=\operatorname{Gen} x_{i-a+b} ; \operatorname{Gen} x_{i-a+b}=\operatorname{Gen} x_{i-a} ; \operatorname{Gen} x_{i=a}=c$

12) $b=b-1$

13) if $b \geq a+1$ go to 7 )

14) $a=a-1$

15) if $a>1$ go to 6)

16) STOP

\section{Computational Experience}

The program was made by MATLAB version 7.9.0.529 (R2009b) 32-bit (win32). To run the program we use laptop with processor Intel (R) core (TM) i3-3217U, CPU $1.80 \mathrm{GHz}$ RAM

$4.00 \mathrm{~GB}$.

\subsection{Esc 16b, Esc 16c, Esc 16h Problem Instances}

The $16 \times 16$ QAP problem adopted from Eschermann and H. J. Wunderlich (1990). To solve the problem we use combination methods, that combines random point strategy, forward exchange strategy and backward exchange strategy, then execute in a numbers of iteration. The result can be seen in table 1 and table 2 below.

Table 1: The Search Table for Esc16 Using Combination Methods

\begin{tabular}{|c|c|c|c|c|c|}
\hline $\begin{array}{l}\text { Problem } \\
\text { Instance }\end{array}$ & $\begin{array}{l}\text { Number of } \\
\text { Iteration }\end{array}$ & Objective Value & \multicolumn{2}{|c|}{ Permutation } & Running Time \\
\hline Esc $16 b$ & 500 & 292 (opt) & \multicolumn{2}{|c|}{23169713151511144128106} & 371.654879 \\
\hline Esc $16 c$ & 500 & 160 (opt) & \multicolumn{2}{|c|}{51615876113492101214113} & 270.088915 \\
\hline Esc $16 \mathrm{~h}$ & 500 & 996 (opt) & \multicolumn{2}{|c|}{16158514910612134311127} & 326.956387 \\
\hline Problem & Objective & \multirow{2}{*}{\multicolumn{2}{|c|}{ Permutation with Combination Methods }} & \multirow{2}{*}{\multicolumn{2}{|c|}{ Permutation from QAPLIB }} \\
\hline Instance & Value & & & & \\
\hline Esc $16 b$ & 292 (opt) & \multicolumn{2}{|c|}{$\begin{array}{c}2316971315151114412810 \\
6\end{array}$} & \multicolumn{2}{|c|}{$\begin{array}{c}637513115241191410128 \\
16\end{array}$} \\
\hline
\end{tabular}




$\begin{array}{cccc}\text { Esc 16c } & 160(\mathrm{opt}) & 516158761134921012141 & 1114101612893136571521 \\ & & 13 & 4 \\ \text { Esc 16h } & 996(\mathrm{opt}) & 1615851491061213431112 & 139101531141612785621\end{array}$

\section{Conclusion}

In this research We've found the other assignment for problem instances Esc16b, Esc16c, and Esc16h from QAPLIB. Both of the permutation have same objective value.

The procedure described here has proved successful in obtaining integer feasible solution to the Quadratic Assignment Problem in relatively short computing times as can be seen from the number iteration needed and the running time.

\section{References}

[1] Ahuja R.K., J.B.Orlin, and A.Tiwari, (2000), - A Descent Genetic Algorithm for the Quadratic Assignment Problem I| , Computers and Operations Research, 27, : 917-934.

[2] Ahyaningsih, F., (2006), - Menyelesaikan Quadratic Assignment Problem Dengan Metode Heuristik Kelayakan II, Thesis S2 Magister Mathematic University of North Sumatera Indonesia,.

[3] Ahyaningsih, F., O. S. Sitompul, (2015), - Developing A Combined Strategy For Solving Quadratic Assignment Problem || , International Journal Of Scientific \& Technology Research, Vol 4 Issue 11, November Edition, ISSN 2277-8616; 297-301.

[4] Battiti, R. and G.Tecchiolli, (1994b), - Simulated annealing and tabu search in the long run: a comparison on QAP Tasks -, Computers and Mathematics with Applications, 28,: 1-8.

[5] Battiti, R. and G.Tecchiolli, (1994a), —The reactive tabu search —, ORSA Journal on Computing 6; 126- 140.

[6] Burkard, R.E. and F.Rendl, (1984), - A thermodynamically motivated simulation procedure for combinatorial optimization problems, ॥ European Journal of Operational Research 17,: 169-174.

[7]Connoly, D.T., - An improved annealing scheme for the QAP \| , European Journal of Operational Research 46,(1990) : $93-100$.

[8] Cung, V.D., T.Mautor, P.Michelon, and A. Tavares, - A scatter search based approach for the quadratic assignment problem $\|$, proceedings of the IEEE International Conference on Evolutionary Computation and Evolutionary Programming (ICEC '97),Indianapolis,(1997): 165170.

[9] Drezner, Z., —A New Genetic algorithm for the Quadratic Assignment Problem —, INFORMS Journal on Computing (2002a).

[10] Drezner, Z., —Robust Heuristic Algorithm for the Quadratic assignment Problem -, EJOR (2002b). Elshafei, A. N., 一Hospital lay-out as a quadratic assignment problem —, Operational Research Quaterly 28, (1977) : 167-179.

[11] Eschermann, B. and H. J. Wunderlich, || Optimized Synthesis of Self-Testable Finite State Machines II , in 20th International Symposium on Fault-Tolerant Computing (FFTCS 20), Newcastle upon Tyne, 26-28th June, (1990).

[12] Fleurent, C. and J.Ferland, -Genetic hybrids for the quadratic assignment problem —, DIMACS Series in Math. Theoritical Computer Science 16,( 1994) : 190-206.

[13] Gambardella, L.M., E.D.Taillard and M.Dorigo, - Ant colonies for the quadratic assignment problem -, Journal of the Operational Society 50,(1999): 167-176.

[14] Laporte, G. and H.Mercure, —Balancing hydraulic turbine runners: A quadratic assignment problem —, EJOR 35, (1998): $378-381$.

[15] Li, Y., P.M.Pardalos, and M.G.C.Resende, - A randomized adaptive search procedure for the quadratic assignment problem $\|$, in Quadratic Assignment and Related Problems II , P.Pardalos and H. Wolcowicz (eds.), DIMACS series in Discrete mathematics and Theoritical Computer Science, 16, (1994): 237-261.

[16] Misevicius, A., and D.Rubliauskas, - Testing of hybrid genetic algorithms for structured Quadratic Assignment Problems I| , Informatica 20, (2009) : 255-272.

[17] Skorin-Kapov, J. (1990)., - Tabu Search Applied to the Quadratic Assignment Problem II , ORSA Journal on Computing 2, $33-45$.

[18] Sondergeld, L. and Voss, - A star-shaped diversification approach in Tabu search - , in: - Meta-heuristics: Theory and Applications II , I.H.Osman and J.P.Kelly (eds.), Kluwer academic Publishers, (1996): 489-502.

[19] Stutzle, T. and H.Hoos, - the Max-Min ant system and local search for combinatorial optimization problems: Towards adaptive Tools for Global Optimization \| :, in : - Meta-heuristics: Advances and Trends in Local Search Paradigms for Optimization II , S.Voss, S.Martello, I.H.Osman, C.Roucairol (eds.), Kluwer academic Publishers, (1999): 313-329.

[20] Taillard, E.D., —Comparison of iterative searches for the quadratic assignment problem —, Location Science 3, (1995) : 87-105.

[21] Taillard, E.D., -FANT:Fast ant system —, Technical report IDSIA, Lugano , (1998) :46-98.

[22] Taillard, E.D., —Robust tabu search for the quadratic assignment problem —, Parallel Computing 17, (1991) : 443-455.

[23] Tate, D.E. and A.E.Smith, - A genetic approach to the quadratic assignment problem —, Computers and Operations Research 1,( 1995): $855-865$. 